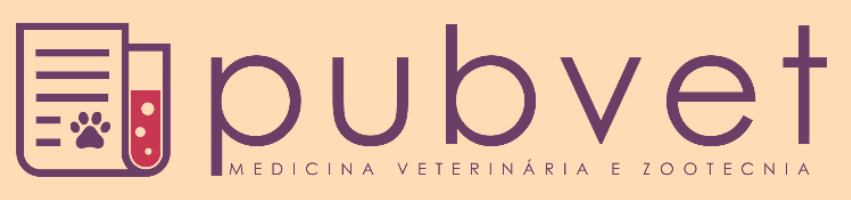

https://doi.org/10.31533/pubvet.v13n11a450.1-8

\title{
Ferramentas de gestão aplicadas à propriedade leiteira: um estudo de caso no município de Rio Branco-Acre
}

\author{
Jaiane Medeiros Vasconcelos ${ }^{1 \bullet}$, Antônia Kaylyanne Pinheiro ${ }^{1 \bullet}$, Eduardo Mitke Brandão \\ Reis $^{2 *} \bullet$, José Marques Carneiro Junior ${ }^{3}$, Maykel Franklin Lima Sales ${ }^{30}$ \\ ${ }^{I}$ Doutoranda da Universidade Federal do Acre, Programa de Pós-graduação em Sanidade e Produção Animal Sustentável na Amazônia \\ Ocidental, Rio Branco Acre, Brasil. \\ ${ }^{2}$ Professor da Universidade Federal do Acre, Centro de Ciências Biológicas e da Natureza, Rio Branco - AC, Brasil. \\ ${ }^{3}$ Pesquisador da Empresa Brasileira de Pesquisa Agropecuária, Rio Branco-AC, Brasil. \\ *Autor para correspondência, E-mail: edumitke@gmail.com
}

Resumo. Objetivou-se analisar a aplicabilidade de ferramentas de gestão em uma propriedade leiteira no estado do Acre, visando identificar os principais pontos fracos e propor soluções no intuito de minimizar perdas zootécnicas e econômicas. Os dados utilizados foram provenientes de uma propriedade, localizadas na região de Rio BrancoAC em maio de 2019, sendo coletados a partir de um formulário semiestruturado, contendo 549 questões. As respostas foram obtidas por meio da observação, bem como por entrevista com o proprietário. Utilizando-se a matriz GUT, os principais pontos fracos encontrados, em ordem decrescente, foram: alta infestação de carrapato, animais de alta produção não adaptados, não realizar exame de tuberculose e não realizar exame no touro de repasse. A partir destes resultados é possível identificar os principais gargalos e traçar metas para melhoria na propriedade.

Palavras chave: Pecuária leiteira, ferramentas de gestão, matriz GUT

\section{Management tools applied to dairy property: a case study in Rio Branco-Acre}

Abstract. The objective was to analyze the applicability of management tools in a dairy farm in the state of Acre, aiming to identify the main weaknesses and propose solutions in order to minimize zootechnical and economic losses. The data used came from a property located in the Rio Branco-AC region in May 2019 and was collected from a semi-structured form containing 549 questions. Responses were obtained through observation as well as by interview with the owner. Using the GUT matrix, the main weaknesses found, in descending order, were: high tick infestation, non-adapted high-production animals, no tuberculosis examination and no pass-through bull examination. From these results it is possible to identify the main bottlenecks and set goals for property improvement.

Keywords: Dairy cattle, management tools, GUT matrix

\section{Herramientas de gestión aplicadas a la propiedad lechera: un estudio de caso en Rio Branco-Acre}

Resumen. Se objetivó analizar la aplicabilidad de las herramientas de gestión en una granja lechera en el estado de Acre, con el objetivo de identificar las principales debilidades y proponer soluciones para minimizar las pérdidas zootécnicas y económicas. Los datos utilizados provenían de una propiedad ubicada en la región de Rio Branco-AC en mayo de 
2019, y se recopilaron de un formulario semiestructurado conteniendo 549 preguntas. Las respuestas se obtuvieron a través de la observación, así como por entrevista con el propietario. Utilizando la matriz GUT, las principales debilidades encontradas, en orden descendente, fueron: alta infestación de garrapatas, animales de alta producción no adaptados, sin examen de tuberculosis y sin examen de toro de transferencia. A partir de estos resultados, es posible identificar los principales cuellos de botella y establecer objetivos para la mejora de la granja.

Palabras clave: Ganado lechero, herramientas de gestión, matriz GUT

\section{Introdução}

A partir do século XX, a produção mundial de leite aumentou mais de $50 \%$ passando de aproximadamente de 19,7 bilhões no ano de 2000 para 35,3 bilhões em 2018 (ANUALPEC, 2019). No Brasil, o leite está entre os seis produtos mais importantes da agropecuária, sendo essencial na geração de emprego e renda para a população (Leira et al., 2018). A região Sul é a maior produtora do país, representando 38\% do total nacional. Durante o mesmo período, o Estado de Minas Gerais permaneceu como maior produtor Brasileiro, seguido Rio Grande do Sul e Paraná (ANUALPEC, 2019). Além disso, o país possui o segundo maior rebanho bovino a nível mundial, atrás apenas da Índia (ANUALPEC, 2019; FAPRI, 2019).

Na região da Amazônia o estado de Rondônia é o principal produtor de leite, com 1, 03 bilhões de litros, seguido pelo Pará, cuja produção foi de 612,8 milhões de litros (ANUALPEC, 2019). A pecuária leiteira no Acre em 2017 produziu 46,4 milhões de litros de leite, sendo uma atividade de grande relevância social na geração de emprego e renda para pequenos e médios produtores da região. Contudo possuem baixo nível tecnológico e animais de baixo padrão genético gerando uma produção média de $5,0 \mathrm{~kg}$ por vaca dia (Cerdótes et al., 2004). Neste sentido as ferramentas de gestão possuem grande importância para auxiliar nas tomadas de decisões da propriedade.

As ferramentas de gestão são técnicas para uso empresarial visando, entre outros, solução de problemas gerenciais, e seu uso pode auxiliar empresários na tomada de decisões dentro de seu processo produtivo, pois os orientam na observação, identificação e análise de problemas (Meireles, 2001). Essa pesquisa tem por objetivo analisar a aplicabilidade de ferramentas de gestão em uma propriedade leiteira no estado do Acre, visando identificar os principais pontos fracos e propor soluções.

\section{Metodologia}

O presente trabalho trata-se de um estudo de caso elaborado em uma propriedade leiteira típica do estado do Acre. $\mathrm{O}$ estudo de caso é um método que permite organização dos dados devido a sua flexibilidade, sendo bastante recomendado nas fases iniciais de uma investigação relacionados a temas complexo, para construção de hipóteses ou reformulação de problema (Triviños, 2015; Yin, 1981). Constitui-se de uma pesquisa exploratória e de caráter qualitativo, visando proporcionar ao pesquisador melhor conhecimento do problema em estudo e, consequentemente, tornar um problema complexo em uma possível solução (Bryman \& Cramer, 2004; Ciribelli, 2003).

A pesquisa foi realizada em um sistema de produção de leite localizado no estado do Acre, cidade de Rio Branco, em maio de 2019, por uma equipe multidisciplinar composta por doutorandas em ciências animal e um professor do Programa de Pós-Graduação. Para escolha da propriedade foi utilizada a amostragem não probabilística por julgamento que levou em consideração os seguintes quesitos: acesso da propriedade, disponibilidade do proprietário, conhecimento e prática sobre o sistema produtivo da sua propriedade.

Para levantamento dos dados, foi realizado um diagnóstico utilizando-se um formulário semiestruturado, com a finalidade de elencar os pontos fracos da propriedade em estudo (Lopes et al., 2016). O formulário é composto por 549 questões sendo 52 questões relacionadas à caracterização do produtor e da propriedade, 12 questões relacionadas à caracterização do rebanho e 485 questões são referentes a caracterização da produção de leite. As questões específicas à caracterização da produção de leite incluem: sistema de produção, práticas agrícolas, manejo nutricional, infraestrutura, escrituração 
zootécnica, identificação dos animais, manejo reprodutivo, qualidade do leite, manejo de ordenha, criação de bezerras, controle sanitário e manejo ambiental.

Após a aplicação do questionário na fazenda, foi realizada uma investigação e identificação dos pontos fracos no sistema de produção da propriedade por meio da ferramenta de gestão brainstorming (Andrade \& Boff, 2014). A avaliação ocorreu individualmente por cada membro da equipe por meio de uma pontuação de zero a cinco, onde pontuação mais estáveis estão próximas a zero e nível mais crítico à medida que aumenta para cinco. Para isto utilizou-se a ferramenta de gestão matriz GUT (Meireles, 2001). Os dados foram transcritos em uma planilha com as respectivas notas e foi estimada uma média para as diferentes notas atribuídas a cada pesquisador e os pontos fracos, a partir da pontuação média, foram ranqueados em ordem decrescente. Com o intuito de indicar a ferramenta de gestão mais adequada para a solução dos pontos fracos foi utilizado novamente à ferramenta brainstorming (Coletti et al., 2010) e selecionou: Diagrama de Ishikawa (Ishikawa, 1985), 5W2H (Veiga et al., 2013) e PDCA (Aguiar, 2006).

\section{Resultados e discussão}

\section{Pontos fracos encontrados no sistema de produção de leite estudado}

Os pontos fracos encontrados durante a realização do diagnóstico, listados em ordem decrescente, pela pontuação atribuída após a utilização da matriz GUT (Meireles, 2001), podem ser observados na tabela 1. Os principais pontos foram: Alta infestação de carrapato, animais de alta produção não adaptados, não realiza exame de tuberculose e não realiza exame no touro de repasse. As maiores pontuações indicam os pontos fracos que deverão ser priorizados na tentativa de equacioná-los ou minimizá-los, pois possuem maior gravidade, maior urgência em ser resolvidos e maiores tendências de se agravar.

Tabela 1. Pontuação atribuída por meio da matriz GUT a cada ponto fraco identificado na propriedade leiteira situada na cidade de Rio Branco no estado do Acre, maio de 2019

\begin{tabular}{lccccc}
\hline Pontos fracos & \multicolumn{2}{c}{ Nota dos Pesquisadores } & \multirow{2}{*}{ Média } & \multirow{2}{*}{$\begin{array}{c}\text { Desvio } \\
\text { Padrão }\end{array}$} \\
\cline { 2 - 3 } & 1 & 2 & 3 & & \\
1. Alta infestação de carrapato & 125 & 48 & 125 & 99,33 & 36,3 \\
2. Animais de alta produção não adaptados & 125 & 48 & 125 & 99,33 & 36,3 \\
3. Não realiza exame de tuberculose & 64 & 64 & 100 & 76 & 16,97 \\
4. Não realiza exame no touro de repasse & 64 & 100 & 64 & 76 & 16,97 \\
5. Cercas em condições ruins & 100 & 48 & 80 & 76 & 21,42 \\
6. Touro de repasse é do vizinho & 64 & 125 & 24 & 71 & 41,53 \\
7. As vacas são secas 5 meses antes do parto & 64 & 48 & 100 & 70,67 & 21,75 \\
8. Curral de espera coberto sem piso com presença de lama & 100 & 27 & 64 & 63,67 & 29,80 \\
9. Bezerras doentes não são separadas & 80 & 27 & 80 & 62,33 & 24,98 \\
10. Não vacina no pré-parto & 64 & 36 & 80 & 60 & 18,18 \\
11. Presença de invasoras na pastagem & 48 & 48 & 80 & 58,67 & 15,08 \\
12. Não realiza análise do leite & 80 & 24 & 60 & 54,67 & 23,17 \\
13. Mesma suplementação para todas as categorias & 36 & 48 & 75 & 53 & 16,31 \\
14. Não tem banco de colosso & 80 & 36 & 27 & 47,67 & 23,16 \\
15. Não utiliza práticas de conservação de forrageira & 64 & 48 & 27 & 46,33 & 15,15 \\
16. Não tem fonte na água perene na propriedade & 64 & 36 & 36 & 45,33 & 13,20 \\
17. Os utensílios são secos ao ar livre & 100 & 12 & 18 & 43,33 & 40,14 \\
18. Ordenha mecânica não instalada & 48 & 60 & 18 & 42 & 17,66 \\
19. Não realiza tratamento de água & 64 & 27 & 18 & 36,33 & 19,91 \\
20. Não realiza biocarapatecidograma & 64 & 27 & 8 & 33 & 23,25 \\
\hline
\end{tabular}

A realização do diagnóstico é fundamental para identificar os pontos fracos, uma vez que tais pontos fracos comprometem o futuro produtivo e econômico do sistema de produção. Os pontos fracos identificados nesse sistema apresentam de média à alta complexidade. Sendo necessária uma 
intervenção rápida afinal a alta infestação de carrapatos e animais de alta produção não adaptados ao sistema causam altos prejuízos produtivos. Com base nos pontos elencados pode-se realizar um plano de ação e metas a serem cumpridas no curto e longo prazo. A coleta de dados, por meio do diagnóstico realizado, visou agilizar as tomadas de decisões dentro do sistema de produção de leite.

O uso das ferramentas de gestão se torna útil dentro da propriedade, atuando na identificação do problema, e ordenando-os conforme sua gravidade, urgência e tendência a piorar, para que possam ser solucionados. Para que isso ocorra, são necessários conhecimentos de gestão para aplicação em fazendas leiteiras, gerados por esta pesquisa. Diante disso, a aplicação de ferramentas poderá abrir caminho para construção de um plano estratégico de gestão, a partir de metodologias de diagnóstico organizacional e sistema de administração, e para consolidar a tomada de decisões, além de contribuir para a capacitação de recursos humanos em administração de empreendimentos rurais.

\section{Primeiro ponto fraco: alta infestação de carrapato}

Os carrapatos causam consideráveis reduções na produção de leite, na taxa de natalidade além de elevar os gastos com carrapaticidas e mão-de-obra utilizados para seu controle (Cordovés, 1997; Godoi \& Silva, 2009; Gomes, 1998). É também vetor dos agentes patogênicos, muitas vezes letais aos bovinos como os que causam o complexo infeccioso conhecido por "Tristeza Parasitária Bovina" (Guglielmone et al., 2006).

As infestações por carrapatos podem reduzir até 90 litros de leite numa lactação de 300 dias de uma vaca. O carrapato ocasiona esse prejuízo principalmente por causa do estresse que causa, pois além das picadas doloridas e do incômodo ocasionado nos animais, também tem a capacidade de transmitir doenças (Grisi et al., 2014). Os níveis das infestações por carrapatos estão relacionados a três fatores principais: taxa de lotação; temperatura versus umidade e raça dos animais. Quanto maior a taxa de lotação, maior é o desafio. As infestações aumentam quando a temperatura média está em $27^{\circ} \mathrm{C}$ e a umidade igual ou maior que 70\%, como presente nessa região do Acre. Os animais taurinos, raças mais utilizadas para sistema leiteiro, são mais suscetíveis do que os zebuínos (Pires, 2010).

\section{Ferramenta proposta para solucionar o primeiro ponto fraco: $5 \mathrm{~W} 2 \mathrm{H}$}

Para a solução do ponto fraco "alta infestação de carrapato", há a necessidade de se tomar a decisão de adotar tal tecnologia e, para tanto, a ferramenta de gestão proposta foi a 5W2H (Lisbôa \& Godoy, 2012), pois esse tipo de decisão não necessita do envolvimento de grande quantidade de pessoas, nem possui complexidade para que possa ser tomada a decisão.

A ferramenta $5 \mathrm{~W} 2 \mathrm{H}$ atua como referência para sustentar as decisões. Dessa forma, permite a realização do acompanhamento, do incremento ou desenvolvimento de um determinado projeto (Oliveira, 1996):

a) What (o que?): Decidir sobre implantar um sistema de controle de carrapato.

b) When (quando?): Imediatamente.

c) Who (quem?): O proprietário do sistema de produção de leite.

d) Where (onde?): Nos animais e na propriedade.

e) Why (por quê?): Melhorar as condições de sanidade animal.

f) How (Como?): Rotação de pastagem, rodízio de princípio ativo.

g) How Much (quanto custa?): Avaliar de acordo com a quantidade de animais e o preço dos carrapaticidas.

\section{Segundo ponto fraco: Animais de alta produção não adaptados}

Animais de alta produção não adaptados ao sistema produtivo pode estar relacionado ao estresse térmico, esse por sua vez reduz a eficiência produtiva e reprodutiva dos animais. Além de gerar distúrbios metabólicos e maiores chances de o animal adoecer (Bilby et al., 2009). Uma das reações fisiológicas mais imediatas ao estresse calórico é a redução no consumo de alimentos, consequentemente ocorre redução na produção e nos constituintes do leite, acarretando prejuízos aos produtores (Dahl et al., 2000). 


\section{Ferramenta proposta para solucionar o segundo ponto fraco: Diagrama de Ishikawa}

Para solucionar o ponto fraco "Animais de alta produção não adaptados" foi proposto o Diagrama de Ishikawa (Ishikawa, 1985), como ferramenta de gestão, devido à quantidade de variáveis envolvidas para a resolução do problema. O Diagrama de Ishikawa, também conhecido como Diagrama de Causa e Efeito ou Diagrama Espinha-de-peixe, é uma ferramenta gráfica utilizada para o gerenciamento e controle da qualidade em processos diversos. Para Ishikawa (1985), a composição do diagrama considera que os problemas podem ser classificados em sete tipos diferentes de causas. São os 7Ms: Machine (Máquina), Method (Método), Mother Nature (Meio Ambiente), Man Power (Mão de Obra), Material (Matéria-prima), Management (Gestão) e Measurement (Medida)).

Em alguns casos pode-se utilizar outro "M", o das Finanças (Money).

Para construir o diagrama, primeiramente, define-se o problema, ou efeito, a ser analisado. Em seguida, pode ser feito um breve Brainstorming para evidenciar a maior quantidade de causas que contribuem para o surgimento do problema; para tanto, basta perguntar o porquê de aquele problema estar acontecendo. $\mathrm{O}$ diagrama de Ishikawa pode ser visualizado na figura 1.

Para que isso ocorra é necessário corrigir problemas secundários, que levam os animais a não se adaptarem, tal como o manejo. Porém, cada problema secundário necessita ter as suas próprias causas resolvidas. Por exemplo, para que o problema secundário Manejo seja solucionado é preciso trabalhar um item fundamental: a equipe envolvida. Caso esse item secundário não seja minimizado, não se conseguirá resolver o ponto fraco principal. Quanto ao problema secundário manejo, ele não pode ser corrigido após a implantação do sistema de produção. A avaliação desse item deve ser feita de forma preventiva, ao se realizar a elaboração do projeto (planejamento) da atividade agropecuária. Este exemplo demonstra o quanto é complexa a resolução do problema primário; porém factível, como proposto na figura 1.

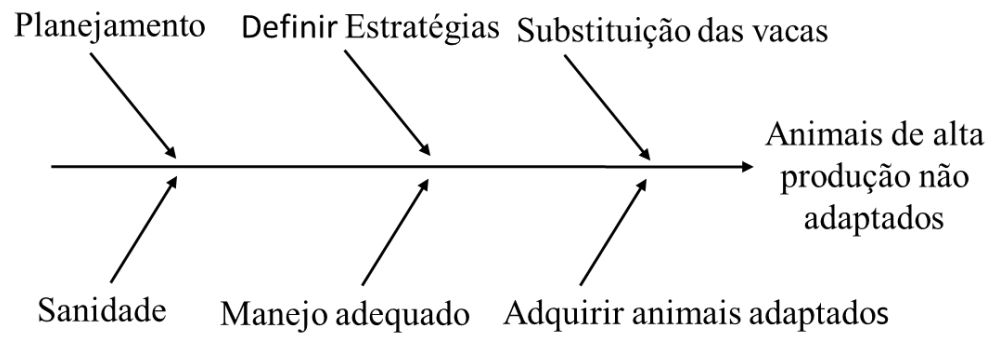

Figura 1. Diagrama de Ishikawa como proposta para resolução do ponto fraco: animais de alta produção não adaptados.

\section{Terceiro ponto fraco: Não realiza exame de tuberculose}

No sistema de produção estudado não foi possível observar métodos de controle sobre doenças infecciosas. A tuberculose e a brucelose são importantes zoonoses de distribuição mundial com grande relevância na bovinocultura (principalmente leiteira) que acarretam prejuízos econômicos e problemas na saúde pública (Mocci et al., 2015). A importância econômica atribuída a essas doenças está baseada nas perdas diretas resultantes da morte de animais, da queda no ganho de peso, diminuição da produção de leite, do descarte precoce e condenação de carcaças no abate (Souza et al., 2014).

A tuberculose é causada pelo Mycobacterium bovis que é um patógeno de importância significativa na pecuária (Delahay et al., 2007; Hermoso de Mendoza et al., 2006). Sabe-se também que pode causar a doença em seres humanos, o que tem levantado preocupações em relação à ocorrência de zoonoses em humanos, especialmente aqueles que vivem na interface homem-animal (Poletto et al., 2004; Ribeiro et al., 2003). A propriedade pesquisada não tem um planejamento de controle à tuberculose bovina, sendo fundamental estimar a prevalência dessa enfermidade, através de exames de diagnóstico, para que seja possível identificar os animais contaminados, e fornecer subsídios para a melhor implementação e gestão do Programa Nacional de Controle e Erradicação de Brucelose e Tuberculose (PNCEBT, 2006), pois os prejuízos causados por elas são enormes. 


\section{Ferramenta proposta para solucionar o terceiro ponto fraco: ciclo PDCA}

A ferramenta de gestão proposta para a minimização do ponto fraco "Não realiza exame de tuberculose" foi a 5W2H (Lisbôa \& Godoy, 2012), esse tipo de decisão não necessita do envolvimento de grande quantidade de pessoas, nem possui complexidade para que possa ser tomada a decisão.

A ferramenta 5W2H atua como referência para sustentar as decisões. Dessa forma, permite a realização do acompanhamento, do incremento ou desenvolvimento de um determinado projeto (Oliveira, 1996).

a) What (o que?): Realizar exame de tuberculose em todo rebanho.

b) When (quando?): Imediatamente.

c) Who (quem?): Um profissional capacitado.

d) Where (onde?): Na própria propriedade.

e) Why (por quê?): Melhorar as condições de sanidade dos animais e da propriedade.

f) How (Como?): Contratando um profissional capacitado.

g) How Much (quanto custa?): Avaliar de acordo com o profissional contratado e os números de animais.

\section{Quarto ponto fraco: Não realiza exame no touro de repasse}

A importância da realização do exame no touro de repasse está relacionado a uma enfermidade conhecida como brucelose, uma doença infectocontagiosa caracterizada por manifestações clínicas da esfera reprodutiva e severos prejuízos aos produtores (Acha \& Szyfres, 2003). Nos touros, a patogenicidade do agente está associada à infecção das glândulas acessórias e aos testículos (Hafez \& Hafez, 2004), manifestada principalmente por vesiculite e, secundariamente, por quadros de orquite e epididimite (Radostits et al., 2010), levando frequentemente os animais infectados a sub e/ou infertilidade (Morrow, 1980).

\section{Ferramenta proposta para solucionar o quarto ponto fraco: Não realiza exame no touro}

Para a solução do ponto fraco "não realiza exame no touro", há a necessidade de se tomar a decisão de adotar tal tecnologia e, para tanto, a ferramenta de gestão proposta para foi a 5W2H (Lisbôa \& Godoy, 2012), pois esse tipo de decisão não necessita do envolvimento de grande quantidade de pessoas, nem possui complexidade para que possa ser tomada a decisão.

A ferramenta $5 \mathrm{~W} 2 \mathrm{H}$ atua como referência para sustentar as decisões. Dessa forma, permite a realização do acompanhamento, do incremento ou desenvolvimento de um determinado projeto (Oliveira, 1996).

a) What (o que?): Decidir sobre fazer exame no touro.

b) When (quando?): Imediatamente.

c) Who (quem?): O proprietário do sistema de produção de leite.

d) Where (onde?): Na propriedade.

e) Why (por quê?): Melhorar as condições de sanidade do animal e da propriedade.

f) How (Como?): Contratando um médico veterinário capacitado.

g) How Much (quanto custa?): Avaliar de acordo com o profissional contratado.

\section{Referências bibliográficas}

Acha, P. N. \& Szyfres, B. (2003). Zoonosis y enfermedades transmisibles comunes al hombre ya los animales: clamidiosis, rickettsiosis y virosis. 3. Washimgton: Pan American Health Organization.

Aguiar, S. (2006). Integração das ferramentas da qualidade ao PDCA e ao Programa 6 Sigma. Nova Lima: Tecnologia e Serviços LTDA., 1205-206.

Andrade, S. P. \& Boff, C. D. S. (2014). Ferramentas de planejamento para tomada de decisão aplicadas a um microempreendedor individual (MEI). Revista de Contabilidade, Ciência da Gestão e Finanças, 2(1):57-85.

ANUALPEC. (2019). Anuário da Pecuária Brasileira (20th ed. Vol. 1). São Paulo, São Paulo, Brasil: Instituto FNP.

Bilby, T. R., Tatcher, W. W. \& Hansen, P. J. (2009). Estratégias farmacológicas, nutricionais e de manejo para aumentar a fertilidade de vacas leiteiras sob estresse térmico. 
Brasil. (2006). Ministério da Agricultura, Pecuária e Abastecimento. Programa nacional de controle e erradicação da brucelose e da tuberculose animal (PNCEBT): manual técnico. MAPA/SDA/DAS.

Bryman, A. \& Cramer, D. (2004). Quantitative data analysis with SPSS 12 and 13: A guide for social scientists. London, UK: Routledge.

Cerdótes, L., Restle, J., Alves Filho, D. C., Nörnberg, M. F. B. L., Nörnberg, J. L., Heck, I. \& Silveira, M. F. (2004). Produção e composição do leite de vacas de quatro grupos genéticos submetidas a dois manejos alimentares no període de lactação. Revista Brasileira de Zootecnia, 33(3):610-622.

Ciribelli, M. C. (2003). Como elaborar uma dissertação de mestrado através da pesquisa científica. São Paulo, Brasil: 7Letras.

Coletti, J., Bonduelle, G. M. \& Iwakiri, S. (2010). Avaliação de defeitos no processo de fabricação de lamelas para pisos de madeira engenheirados com uso de ferramentas de controle de qualidade. Acta Amazonica, 40(1):135-140.

Cordovés, C. O. (1997). Carrapato: controle ou erradicação (Vol. 1). Porto Alegre, Rio Grande do Sul: Guaíba Agropecuária.

Dahl, G. E., Buchanan, B. A. \& Tucker, H. A. (2000). Photoperiodic Effects on Dairy Cattle: A Review. Journal of Dairy Science, 83(4):885-893. doi: http://dx.doi.org/10.3168/jds.S0022-0302(00)74952-6

Delahay, R. J., Smith, G. C., Barlow, A. M., Walker, N., Harris, A., Clifton-Hadley, R. S. \& Cheeseman, C. L. (2007). Bovine tuberculosis infection in wild mammals in the South-West region of England: A survey of prevalence and a semi-quantitative assessment of the relative risks to cattle. The Veterinary Journal, 173(2):287-301. doi: http://dx.doi.org/10.1016/j.tvjl.2005.11.011

FAPRI. (2019). Food and Agricultural Policy Research Institute. Food and Agricultural Policy Research Institute, from http://www.fapri.iastate.edu/tools/outlook.aspx

Godoi, C. R. \& Silva, E. F. P. (2009). Carrapato Boophilus microplus e impacto na produção animalRevisão de literatura. PUBVET, 3(22):Art 606.

Gomes, A. (1998). Controle do carrapato do boi: um problema para quem cria raças européias. Embrapa Gado de Corte, 31(1):1-8.

Grisi, L., Leite, R. C., Martins, J. R. d. S., Barros, A. T. M. d., Andreotti, R., Cançado, P. H. D., . . . Villela, H. S. (2014). Reassessment of the potential economic impact of cattle parasites in Brazil. Revista Brasileira de Parasitologia Veterinária, 23(2):150-156.

Guglielmone, A. A., Beati, L., Barros-Battesti, D. M., Labruna, M. B., Nava, S., Venzal, J. M., . . . González-Acuña, D. (2006). Ticks (Ixodidae) on humans in south america. Experimental \& Applied Acarology, 40(2):83-100.

Hafez, B. \& Hafez, E. (2004). Reprodução Animal (Vol. 1, pp. 513): Manole: São Paulo, Brasil.

Hermoso de Mendoza, J., Parra, A., Tato, A., Alonso, J. M., Rey, J. M., Peña, J., . . . Hermoso de Mendoza, M. (2006). Bovine tuberculosis in wild boar (Sus scrofa), red deer (Cervus elaphus) and cattle (Bos taurus) in a Mediterranean ecosystem (1992-2004). Preventive Veterinary Medicine, 74(2-3):239-247. doi: http://dx.doi.org/10.1016/j.prevetmed.2005.10.005

Ishikawa, K. (1985). Controle de qualidade total: à maneira japonesa. Rio de Janeiro, Brasil: Campus.

Leira, M. H., Botelho, H. A., Barreto, B. B., Botelho, J. H. V. \& Pessoa, G. O. (2018). Fatores que alteram a produção e a qualidade do leite: Revisão. PUBVET, 12(5):1-13.

Lisbôa, M. d. G. P. \& Godoy, L. P. (2012). Aplicação do método 5W2H no processo produtivo do produto: a joia. Iberoamerican Journal of Industrial Engineering, 4(7):32-47.

Lopes, M. A., Reis, E. M. B. \& Ferrazza, R. (2016). Formulário de diagnóstico da propriedade leiteira. Lavras, Minas Gerais, Brasil. . Lavras: Universidade Federal de Lavras.

Meireles, M. (2001). Ferramentas administrativas para identificar observar e analisar problemas (Vol. 2). São Paulo: Arte \& Ciência.

Mocci, D., Renesto, D. M., Rodrigues, A. R. A., Silva, R. S. \& Machado, J. G. (2015). Prevalência da brucelose e tuberculose bovina em propriedades da região de São José do Rio Preto-SP. Ars Veterinaria, 30(2):100-103. 
Morrow, D. A. (1980). Current therapy in theriogenology: diagnosis, treatment, and prevention of reproductive diseases in animals. Philadelphia, USA: Saunders.

Oliveira, S. T. (1996). Ferramentas para o aprimoramento da qualidade. São Paulo, Brasil: Editora Pioneira.

Pires, A. V. (2010). Bovinocultura de Corte (Vol. 1). Piracicaba, São Paulo: FEALQ.

Poletto, R., Kreutz, L. C., Gonzales, J. C. \& Barcellos, L. J. G. (2004). Prevalência de tuberculose, brucelose e infecções víricas em bovinos leiteiros do município de Passo Fundo, RS. Ciência Rural, 34(2):595-598.

Radostits, O. M., Gay, C. C., Blood, D. C., Hinchcliff, K. W. \& McKenzie, R. A. (2010). Clínica Veterinária: um tratado de doenças dos bovinos, ovinos, suínos, caprinos e eqüinos (Vol. 1). Rio de Janeiro: Guanabara Koogan.

Ribeiro, A. R. P., Lobato, F. C. F., Abreu, V. L. V., Faria, E. S. \& Silva, J. A. (2003). Prevalência de tuberculose e brucelose bovina no município de Ilhéus. Arquivo Brasileiro de Medicina Veterinária e Zootecnia, 55(1):120-122.

Souza, A. C., Andrade, L. S. S. \& Sousa, D. (2014). Orientações para o descarte responsável de medicamentos de uso veterinário. Jornada de Ensino, Pesquisa e Extensão, 13161-163.

Triviños, A. N. S. (2015). Introdução à pesquisa em ciências sociais: a pesquisa qualitativa em educação. O positivismo; a fenomenologia; o marxismo. São Paulo: Atlas.

Veiga, R. S., Polacinski, É., Silva, V. B., Tauchen, J. \& Pires, M. R. (2013). Implantação dos 5Ss e proposição de um SGQ para uma indústria de erva-mate. Revista ADMPG, 6(1):71-78.

Yin, R. K. (1981). The case study as a serious research strategy. Knowledge, 3(1):97-114.

Recebido: 8 de agosto, 2019.

Aprovado: 1 de novembro, 2019.

Publicado: 16 de dezembro, 2019.

Licenciamento: Este artigo é publicado na modalidade acesso aberto sob a licença Creative Commons Atribuição 4.0 (CC-BY 4.0), a qual permite uso irrestrito, distribuição, reprodução em qualquer meio, desde que o autor e a fonte sejam devidamente creditados. 